\title{
NEUROENDOCRINE CARCINOMA OF URINARY BLADDER: EXTREMELY A RARE ENTITY
}

\author{
MD ABDUR RAKIB ${ }^{1}$, MD SHAHIDUL ISLAM ${ }^{2}$, S M SHAMEEM WAHEED ${ }^{3}$
}

\begin{abstract}
Neuroendocrine carcinoma or small cell carcinoma (SCC) of the bladder is a rare and aggressive tumor associated with a poor prognosis. It often presents at a later stage than urothelial carcinoma of the bladder, and comprises less than $1 \%$ of bladder malignancies. A number of treatment algorithms have been used to treat bladder SCC, including cystectomy, partial cystectomy, radiotherapy, chemoradiotherapy, chemotherapy alone, and neoadjuvant/adjuvant chemotherapy. Here we are presenting a case of, small cell variety of neuroendocrine carcinoma of the bladder, and its epidemiology, prognosis, and current treatment algorithms are reviewed.
\end{abstract}

Key Words: Bladder cancer, Neuroendocrine tumors, Small cell carcinoma

Bangladesh J. Urol. 2019; 22(2):

\section{Introduction}

Small cell carcinoma (SCC) of the bladder is a rare and aggressive tumor associated with a poor prognosis. It often presents at a later stage than urothelial carcinoma of the bladder, and comprises less than $1 \%$ of bladder malignancies. A number of treatment algorithms have been used to treat bladder SCC, including cystectomy, partial cystectomy, radiotherapy, chemoradiotherapy, chemotherapy alone, and neoadjuvant/adjuvant chemotherapy. Presented is a case of SCC of the bladder, and the epidemiology, prognosis, and current treatment algorithms for patients with bladder SCC are reviewed.

In 2008 , the incidence of bladder cancer was 68,810 cases per year in the United States[1]. It is 3 times more common in men than in women. In men, it is the fourth most common malignancy after prostate, lung, and colorectal cancers[2]. In women, bladder cancer is the ninth most common cancer. More than $90 \%$ of bladder cancers are urothelial carcinomas. The two most common nonurothelial epithelial malignancies of the bladder are squamous cell carcinoma and

1. Urology Centre, Combined Military Hospital Dhaka, Dhaka Cantonment

2. Head of the department of Urology Centre, Combined Military Hospital Dhaka, Dhaka Cantonment

3. Urology Centre, Combined Military Hospital Dhaka, Dhaka Cantonment

Correspondence: Lt Col (Associate Professor) Md Abdur Rakib, Urology Centre, Combined Military Hospital Dhaka, Dhaka Cantonment, Mob-01769014706, 01720955484

Received: 05 February 2019

Accepted: 10 May 2019 adenocarcinoma. Neuroendocrine tumors are less common than the above histologic variants in the genitourinary system, and are classified into two subtypes: carcinoid tumor and neuroendocrine carcinoma. Neuroendocrine carcinoma is further subdivided into small cell carcinoma (SCC) and large cell neuroendocrine carcinoma, the latter of which is exceedingly rare in the bladder[3]. SCC of the bladder comprises only $0.5 \%$ to $1.0 \%$ of primary bladder malignancies. It most commonly presents in the seventh decade, with a male: female ratio of $2: 1$ to $5: 1$. Unlike lung SCC, bladder SCC rarely is associated with paraneoplastic syndromes. Pure SCC of the bladder is infrequent and is usually mixed with another histologic subtype (most commonly urothelial carcinoma) [3]. SCC of the bladder usually behaves more aggressively than urothelial carcinoma and carries a worse prognosis, often because patients present at a later stage as compared with urothelial carcinoma[4]. The bladder is the most common site for genitourinary extrapulmonary SCC[5]. We describe a case of neuroendocrine carcinoma of the urinary bladder presenting with gross hematuria in a 52-year male.

\section{Case Report}

Mr Shah Jalal, aged 52 yrs from Comilla presented with 3 episodes of painless total gross heamatruria within last (April 2018) one month associated with anorexia but no wt loss. He is a smoker but non diabetic and normotensive, no other co-morbidity. His physical 
Neuroendocrine Carcinoma of Urinary Bladder: Extremely A rare Entity

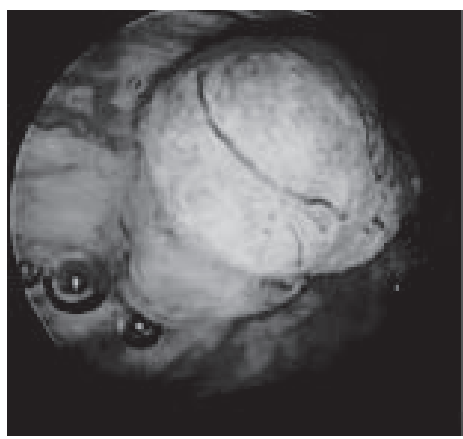

Fig.-1: Cystoscopic view of tumour

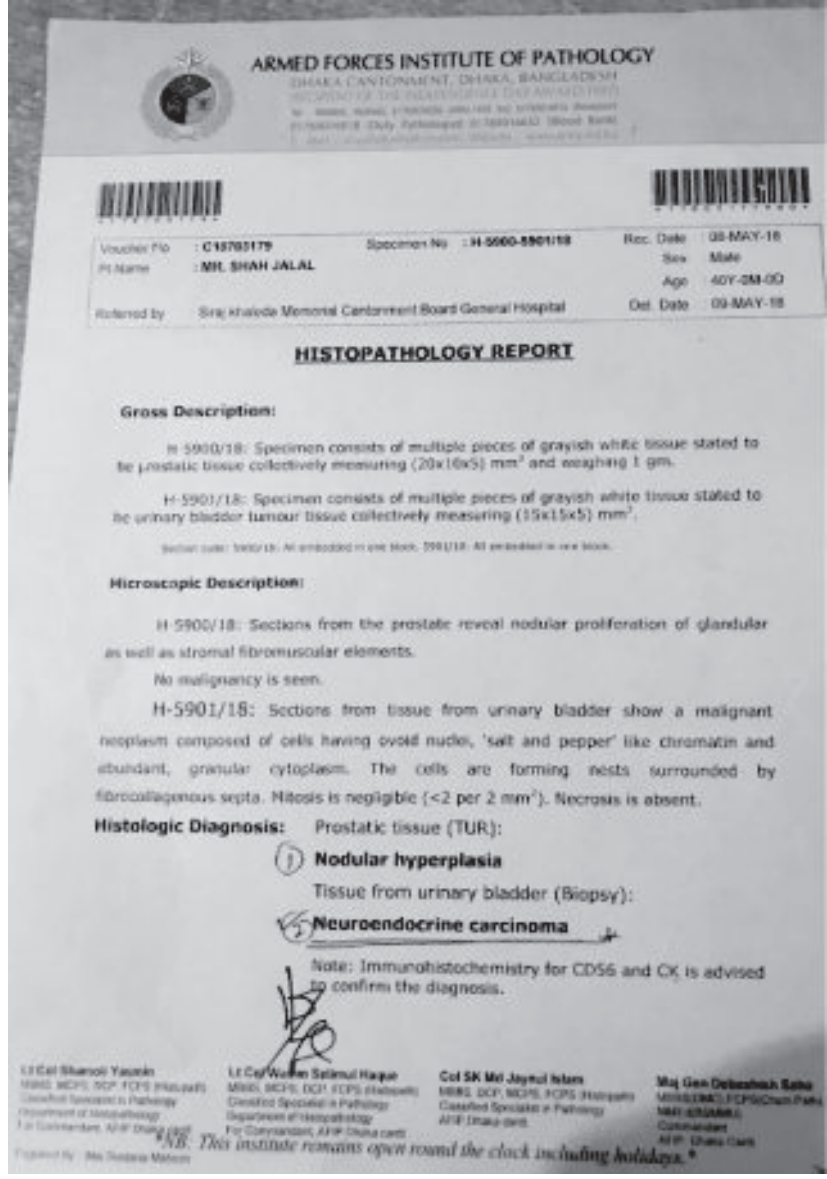

examination is unremarkable. All the bio-Chemical and heamatological parameters are normal except microscopic heamaturia. Ultrasonography of KUB shows a nodular soft tissue mass arising from base of urinary bladder close to the left ureteric orifice measuring $5.4 \times 3.2 \mathrm{~cm}$. Urethrocystoscopy shows a large solid, polypoid, angry looking growth at the base of urinary bladder with multiple dilated angry looking blood vessels around the tumour.

He underwent transurethral resection of the bladder tumour (TURBT) for pathological diagnosis.
Fig.-2: Histopathology of tumour

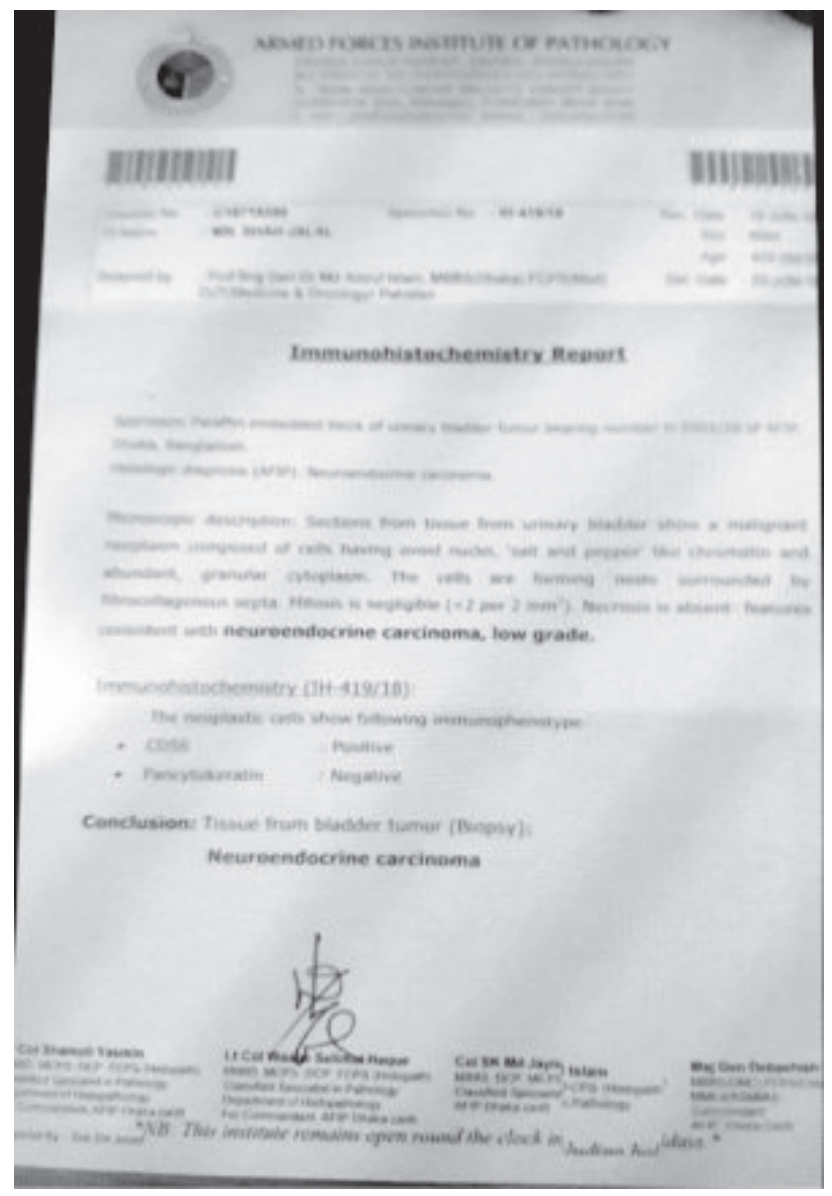

Transurethral resection of bladder tumour (TURBT) specimen shows a malignant tumour composed of cells having ovoid nuclei, 'salt and pepper' like chromatin and abundant granular cytoplasm. Immunohistochemistry for CD56 is positive which confirm as neuroendocrine carcinoma. The patient was discharged on $4^{\text {th }}$ post operative day and advised for regular follow up.

\section{Discussion}

As with urothelial carcinoma, patients with SCC can present with dysuria, obstructive voiding symptoms, 
weight loss, abdominal pain, ureteral obstruction, and/ or recurrent UTIs. ${ }^{4}$ In both urothelial carcinoma and SCC of the bladder, the most common presentation is painless hematuria $(67 \%-100 \%$ in bladder SCC) [6]. Cigarette smoking is a risk factor for SCC of the bladder with $50 \%$ to $70 \%$ of patients reporting a smoking history. ${ }^{6}$ Bladder SCC more often presents at a later stage than urothelial carcinoma. As such, worse outcomes are associated with SCC of the bladder. Bladder SCC presents as stage I in $0 \%$ to $5 \%$ of patients, stage II in $27 \%$ to $44 \%$ of patients, stage III in $24 \%$ to $30 \%$ of patients, and stage IV in $27 \%$ to $43 \%$ of patients[6]. Median survival of all those with bladder SCC is 20 to 23 months. ${ }^{4}$ Prognosis is dependent on performance status and extent of disease at diagnosis, whereas overexpression of p53 gene, patient age, gender, and presenting symptoms do not appear to correlate with prognosis. ${ }^{6}$ In addition, it has been found that pure SCC tends to have a poorer outcome than mixed SCC of the bladder[7].

At cystoscopy, SCC cannot be differentiated from urothelial carcinoma by its morphologic appearance[5]. Diagnosis is confirmed with transurethral resection for tissue sampling. Histologically, the diagnosis is supported by positive immunostaining for keratin Cam 5.2 , synaptophysin, and chromogranin. Chromogranin is positive in one-half of the cases of SCC but only $5 \%$ of urothelial carcinoma[5]. Also, $40 \%$ of bladder SCCs express thyroid transcription factor[4]. Treatment is dependent on tumor stage at presentation and patient performance status. The low incidence of SCC of the urinary bladder has made it difficult to establish definitive treatment algorithms. Cystectomy, partial cystectomy, transurethral resection, and radiotherapy have all been used to treat local disease[6]. Neoadjuvant and adjuvant chemotherapy have been used with these modalities as well as chemotherapy alone[6]. TURBT alone is usually not curative and is associated with survival rates of 3 to 6 months[6]. TURBT alone is reserved for patients not able to tolerate more aggressive therapies or for palliation of symptoms. Individuals who undergo TURBT with radiotherapy have been reported to have a median survival of 5 to 6.5 months in small, retrospective case series[6]. Surgery alone (cystectomy or partial cystectomy) is reserved for early stage blad der SCC (stage I and II) [6]. Longterm survival has een reported for stage II disease. Choong and collea ues[8] reported cure in 6 of $8(75 \%)$ patients with stage II SCC treated with radical cystectomy alone in a retrospective review of 44 patients from 1975 to 2003.

Neoadjuvant or adjuvant cisplatinum- based chemotherapy has been used with radical cystectomy to potentially improve long-term survival in bladder SCC[6]. In a retrospective review of 25 patients with neuroendocrine tumor of the bladder, Quek and associates[9] reported significant improvement in recurrence-free and overall survival (OS) in those receiving neoadjuvant or adjuvant chemotherapy with radical cystectomy as compared with radical cystectomy alone. Siefker-Radtke and colleagues[10] reported similar results with regard to neoadjuvant chemotherapy but not with adjuvant chemotherapy. In a retrospective review of 88 patients, individuals treated with neoadjuvant chemotherapy had $78 \%$ diseasespecific 5-year survival rates as compared with only $36 \%$ among those treated with cystectomy alone. In contrast, there was no survival advantage conferred with adjuvant chemotherapy compared with cystectomy alone. Neoadjuvant therapy may be advantageous in this patient population compared with adjuvant therapy because a large number of patients with SCC exhibit rapid growth rates making complete resection difficult or impossible secondary to rapidly progressive local disease[10]. Other studies, however, have shown no survival benefit between cystectomy and multimodal therapy. For example, Cheng and colleagues ${ }^{11}$ reported no survival benefit in 64 cases of SCC in those undergoing cystectomy alone compared with multimodal treatment. Choong and coauthors[8] recommend no adjuvant chemotherapy for stage I and II disease after reporting a $75 \%$ cure rate with radical cystectomy alone for stage II disease.

Based on treatment algorithms for SCC of the lung, some clinicians have developed combined chemotherapy/ radiotherapy/TURBT regimens as a bladder-sparing alternative to cystectomy[6]. It is difficult to judge the most optimum local therapy, as there are no direct comparisons between radiotherapy and cystectomy alone. ${ }^{5}$ Lohrisch and colleagues[12] retrospectively reviewed 14 cases of bladder SCC treated with combined chemoradiation in patients with primarily stage III or less disease. They observed a 70\% 2-year survival and 44\% 5-year OS in these individuals. Cisplatin based chemotherapy was the primary chemotherapeutic regimen used[12]. Bex and colleagues[13] also reported success with a bladder-sparing approach, with $64.7 \%(n=11)$ of patients exhibiting a clinical complete response, 8 with 


\section{2(2) 2019}

Neuroendocrine Carcinoma of Urinary Bladder: Extremely A rare Entity

chemoradiotherapy and 3 with transurethral resection (TUR) and radiotherapy. Indeed, platinum-based chemotherapy is the mainstay of treatment of recurrent and metastatic disease[7,14]. Mackey and associates[15]. retrospectively reviewed the records of 180 patients with genitourinary SCC. Patient age, sex, primary site, histologic features, tumor size, stage, locoregional therapy, systemic chemotherapy, and hormonal therapy were analyzed for association with survival. For bladder SCC, only cisplatinbased chemotherapy predicted survival on multivariate analysis[15]. One of the drawbacks of chemoradiotherapy is the occurrence of urothelial carcinoma of the bladder, which has been shown to occur in $20 \%$ to $60 \%$ of patients[12,26].

\section{Conclusion}

Bladder SCC usually presents at a later stage than urothelial carcinoma and therefore confers a worse prognosis. Multiple treatment algorithms have been used to treat local and metastatic disease, including cystectomy, partial cystectomy, radiation therapy, and neoadjuvant/adjuvant chemotherapy as well as chemotherapy alone. Low disease incidence makes it difficult to exclusively advocate for one treatment algorithm. Prospective studies need to be done to elucidate the most effective treatment by stage for bladder SCC.

\section{References}

1. Jemal A, Siegel R, Ward E, et al. Cancer statistics, 2008. CA Cancer J Clin. 2008;58:71-96.

2. Jemal A, Murray T, Ward E, et al. Cancer statistics, 2005. CA Cancer J Clin. 2005;55:10-30.

3. Mazzucchelli R, Morichetti D, Lopez-Beltran A, et al. Neuroendocrine tumours of the urinary system and male genital organs: clinical significance. BJU Int. 2009;103:1464-1470.

4. Naturale RT, Maclennan GT. Small cell carcinoma of the bladder. J Urol. 2006;176:781.

5. Mukesh M, Cook N, Hollingdale AE, et al. Small cell carcinoma of the urinary bladder: a 15 -year retrospective review of treatment and survival in the Anglian Cancer Network. BJU Int. 2009;103:747-752.
6. Church DN, Bahl A. Clinical review-small cell carcinoma of the bladder. Cancer Treat Rev. 2006;32:588-593.

7. Ismaili N, Heudel PE, Elkarak F, et al. Outcome of recurrent and metastatic small cell carcinoma of the bladder. BMC Urol. 2009;9:4.

8. Choong NW, Quevedo JF, Kaur JS. Small cell carcinoma of the urinary bladder. The Mayo Clinic experience. Cancer. 2005;103:1172-1178.

9. Quek ML, Nichols PW, Yamzon J, et al. Radical cystectomy for primary neuroendocrine tumors of the bladder: the University of Southern California experience. J Urol. 2005;174:93-96.

10. Siefker-Radtke AO, Dinney CP, Abrahams NA, et al. Evidence supporting preoperative chemotherapy for small cell carcinoma of the bladder: a retrospective review of the M. D. Anderson cancer experience. J Urol. 2004; 172:481-484.

11. Cheng L, Pan CX, Yang XJ, et al. Small cell carcinoma of the urinary bladder: a clinicopathologic analysis of 64 patients. Cancer. 2004;101:957-962.

12. Lohrisch C, Murray N, Pickles T, Sullivan L. Small cell carcinoma of the bladder: long term outcome with integrated chemoradiation. Cancer. 1999; 86:2346-2352.

13. Bex A, Nieuwenhuijzen JA, Kerst M, et al. Small cell carcinoma of bladder: a single-center prospective study of 25 cases treated in analogy to small cell lung cancer. Urology. 2005;65:295299.

14. Asmis TR, Reaume MN, Dahrouge S, Malone S. Genitourinary small cell carcinoma: a retrospective review of treatment and survival patterns at The Ottawa Hospital Regional Cancer Center. BJU Int. 2006;97:711-715.

15. Mackey JR, Au HJ, Hugh J, Venner P. Genitourinary small cell carcinoma: determination of clinical and therapeutic factors associated with survival. J Urol. 1998;159:1624-1629.

16. Bastus R, Caballero JM, González G, et al. Small cell carcinoma of the urinary bladder treated with chemotherapy and radiotherapy: results in five cases. Eur Urol. 1999;35:323-326. 